\title{
BMJ
}

\section{Longlasting insecticidal nets for prevention of Leishmania donovani infection in India and Nepal: paired cluster randomised trial}

\author{
Albert Picado, epidemiologist, ${ }^{1}$ Shri Prakash Singh, reader, ${ }^{2}$ Suman Rijal, professor, ${ }^{3}$ Shyam Sundar, \\ professor, ${ }^{2}$ Bart Ostyn, researcher/medical doctor, ${ }^{5}$ François Chappuis, medical doctor, ${ }^{4}$ Surendra Uranw, \\ PhD student, ${ }^{3}$ Kamlesh Gidwani, PhD student, ${ }^{2}$ Basudha Khanal, researcher, ${ }^{3}$ Madhukar Rai, professor, ${ }^{2}$ \\ Ishwari Sharma Paudel, researcher, ${ }^{3}$ Murari Lal Das, professor, ${ }^{3}$ Rajiv Kumar, PhD student, ${ }^{2}$ Pankaj \\ Srivastava, PhD student, ${ }^{2}$ Jean Claude Dujardin, professor, ${ }^{5}$ Veerle Vanlerberghe, researcher, ${ }^{5}$ Elisabeth \\ Wreford Andersen, statistician, ${ }^{1}$ Clive Richard Davies, professor, ${ }^{1}$ Marleen Boelaert, professor ${ }^{5}$
}

'London School of Hygiene and Tropical Medicine, London WC1E 7HT, UK

${ }^{2}$ Banaras Hindu University, Varanasi 221005, India

${ }^{3} \mathrm{BP}$ Koirala Institute of Health Sciences, PO Box 7053, Kathmandu, Nepal

${ }^{4}$ Geneva University Hospitals, Geneva, Switzerland

${ }^{5}$ Institute of Tropical Medicine, 2000 Antwerp, Belgium

Correspondence to: M Boelaert mboelaert@itg.be

Cite this as: BMJ 2010;341:c6760 doi:10.1136/bmj.c6760

\section{ABSTRACT}

Objective To test the effectiveness of large scale distribution of longlasting nets treated with insecticide in reducing the incidence of visceral leishmaniasis in India and Nepal. Design Paired cluster randomised controlled trial designed to detect a $50 \%$ reduction in incidence of Leishmania donovani infection.

Setting Villages in Muzaffarpur district in India and Saptari, Sunsari, and Morang districts in Nepal.

Participants 13 intervention and 13 control clusters. 12691 people were included in the analysis of the main outcome (infection), and 19810 were enrolled for the secondary (disease) end point.

Intervention Longlasting insecticidal nets (treated with deltamethrin) were distributed in the intervention clusters in December 2006.

Main outcome measures Infection was determined by direct agglutination test at 12 and 24 months after the intervention in those who had negative results (titre $\langle 1: 1600)$ at baseline. The effect estimate was computed as the geometric mean of the risk ratios for seroconversion for each cluster pair (net/no net), with its $95 \%$ confidence interval. Formal tests of effect of no intervention were obtained with a paired $t$ test. Results There was no significant difference in the risk of seroconversion over 24 months in intervention (5.4\%; $347 / 6372)$ compared with control (5.5\%; 345/6319 people) clusters (risk ratio $0.90,95 \%$ confidence interval 0.49 to 1.65$)$ nor in the risk of clinical visceral leishmaniasis $(0.99,0.46$ to 1.40$)$. Adjustment for covariates did not alter these conclusions.

Conclusions There is no evidence that large scale distribution of longlasting insecticidal nets provides additional protection against visceral leishmaniasis compared with existing control practice in the Indian subcontinent. The observed effect was small and not significant, though the confidence intervals did not exclude a $50 \%$ change in either direction. Trial registration Clinical Trials NCT 2005-015374.

\section{INTRODUCTION}

Visceral leishmaniasis is a neglected infectious disease ${ }^{1}$ caused by intramacrophage protozoa of the Leishmania donovani complex transmitted by phlebotomine sandflies. Only a fraction of people infected develop clinical visceral leishmaniasis, characterised by febrile splenomegaly, pancytopenia, and progressive wasting, which can be fatal if left untreated. Visceral leishmaniasis, also known as kala-azar, affects an estimated 500000 people annually, mostly in Asia and East Africa, ${ }^{2}$ and is especially prevalent in poor communities. ${ }^{3}$ In India, Nepal, and Bangladesh, it is transmitted between people by Phlebotomus argentipes, ${ }^{4}$ a peridomestic species of sandfly whose indoor biting rhythm peaks at night. ${ }^{5}$ The current vector control strategy is based on indoor residual spraying with dicophane (DDT) or pyrethroids and has been criticised for being costly, not easily accepted, and not sustainable. ${ }^{67}$ Bed nets treated with insecticide, specifically longlasting nets treated with insecticide, have been proposed as an alternative to indoor residual spraying. ${ }^{6}$ Treated nets proved effective in control of malaria ${ }^{8}$ and cutaneous leishmaniasis in Iran and Syria. ${ }^{6}$ Current evidence on effectiveness of treated nets for prevention of visceral leishmaniasis is limited to entomological data ${ }^{6-11}$ and an observational study in Sudan, ${ }^{12}$ but the latter cannot be extrapolated to the Indian subcontinent because the vector is different. Longlasting insecticidal nets have potential for control of visceral leishmaniasis in this region given the behaviour of $P$ argentipes, ${ }^{5}$ the high acceptability of bed nets, ${ }^{13}$ the fact that untreated nets seem to provide some degree of personal protection, ${ }^{14-16}$ and that village-wide distribution of longlasting insecticidal nets reduced the indoor density of Pargentipes. ${ }^{10}{ }^{11}$ In 2005, a community intervention trial was set up in India and Nepal to test the effectiveness of comprehensive coverage with longlasting insecticidal nets on the incidence of $L$ donovani infection in the current context, where 
indoor residual spraying and untreated nets are irregularly used.

\section{METHODS}

Study design

We used a pair matched cluster randomised trial design as visceral leishmaniasis has a patchy spatial distribution and clusters strongly within villages at hamlet/neighbourhood (tola) and household level. Hamlets were chosen as the unit of analysis as they would be the unit of intervention for a control programme. Clusters were paired on the basis of incidence of visceral leishmaniasis between 2003 and 2005 .

\section{Study population}

The trial was conducted in Muzaffarpur district in India and Saptari, Sunsari, and Morang districts in Nepal. The clusters - either a complete hamlet or a ward (that is, the smallest administrative subdivision) - were selected in a two step procedure. In February 2006 we identified 34 clusters with a high number of reported cases of visceral leishmaniasis (22 in India, 12 in Nepal) and conducted a house to house survey to retrospectively estimate the incidence of visceral leishmaniasis between 2003 and 2005. In May 2006, we selected and included in the trial 26 high incidence clusters out of these 34 (16 in India, 10 in Nepal) based on the following criteria: at least one case of visceral leishmaniasis in 2003, 2004, and 2005, indicating continuous $L$ donovani transmission; a minimum $0.8 \%$ average annual incidence rate of visceral leishmaniasis from 2003 to 2005; a population ranging from 350 to 1500 people; and a minimum distance of $1 \mathrm{~km}$ between clusters. A census was conducted in July-August 2006 registering individual and household demographic information. All data were entered into a geo-referenced database. All individuals living for at least six months a year in the clusters were eligible, but blood sampling was restricted to individuals aged over 2 years.

\section{Intervention}

Between November and December 2006, longlasting insecticidal nets (PermaNet 2.0, Vestergaard-Frandsen, Denmark; 75 denier, 25 holes $/ \mathrm{cm}^{2}$, with deltamethrin $\left(55 \mathrm{mg} / \mathrm{m}^{2}\right)$ coated fibres) were distributed in the intervention clusters. Enough nets were provided to protect all household members, taking into account the number of people (median five people/household), their age, sex, and sleeping arrangements (mean 2.5 treated nets/household). Any untreated nets were exchanged for treated nets. Community volunteers promoted the correct use of treated nets by information leaflets and home visits. The control clusters were allowed to continue using any existing conventional strategies for personal protection. They were not provided with treated nets nor was the use of untreated nets promoted. Individual use of nets was monitored during quarterly house to house surveys. For ethical reasons we did not interfere with ongoing indoor residual spraying activities, but we collected and considered information on spraying in the analysis. Free diagnosis and treatment of visceral leishmaniasis was provided in all clusters.

\section{Sample size}

The sample size was calculated with the method for pair matched randomised controlled trials described by Hayes and Bennett. ${ }^{17}$ We aimed to show a $50 \%$ reduction in infection incidence rates in the intervention group compared with control. To determine the number of clusters required per arm we assumed a $2 \%$ yearly $L$ donovani infection incidence rate; 500 inhabitants per cluster with an anticipated $10 \%$ loss to follow-up, $10 \%$ aged below 2 years, and 10\% serologically positive at baseline; and a coefficient of variation between clusters $(\kappa)$ of 0.25 . Under those assumptions we anticipated a power of $90 \%$ with an $\alpha$ risk of $5 \%$. The sample size calculations determined a minimum of 10 clusters per arm. The final number of clusters was increased to 13 per arm because the incidence of visceral leishmaniasis in Nepal during the preparation phase was lower than expected. ${ }^{18}$

\section{Randomisation and allocation}

The 26 clusters were stratified by country (16 in India and 10 in Nepal) and population size (six and four, respectively, having over 710 residents) and then paired by previous average incidence rate of visceral leishmaniasis. Clusters in each pair were randomly allocated to group 1 or 2 . The random selection of clusters into groups was undertaken in Excel (Microsoft), and the difference in the total number of cases of visceral leishmaniasis reported in the past three years between group 1 and 2 had to be less than 10\%. The intervention was then randomly allocated to one of the groups by tossing a coin in the presence of observers.

\section{Outcomes}

The primary outcome of the clinical trial was the num ber of incident $L$ donovani infections as measured by seroconversion with the direct agglutination test at 12 and 24 months after the intervention, NovemberDecember 2007 and 2008, respectively. Serum samples were diluted from 1:400 to 1:25600, and the cut off for positivity on the direct agglutination test was set at a titre of $1: 1600$. This titre, which is lower than the one used for diagnosis of visceral leishmaniasis in clinically suspected cases (1:3200), was chosen to increase the sensitivity to detect $L$ donovani infection. ${ }^{1619}$ The sensitivity and specificity of the direct agglutination test to detect visceral leishmaniasis (disease) were estimated as $94.8 \%$ and $98.7 \%$, respectively, in endemic communities. ${ }^{20}$ Seroconversion was considered only in individuals who had negative results on the direct agglutination test $(\leq 1: 800)$ in the baseline survey (or their first blood sample). A person was considered as a seroconverter if the titre at one of the two subsequent visits was $\geq 1: 1600$ and at least 2 titres above the baseline value. A minimum of 2 titres difference was 
required to take into account the known interobserver variability in direct agglutination test reading $(+1 /-1$ titre $) .{ }^{21}$

All individuals aged over 2 years provided a capillary blood sample collected by finger prick on a Whatman No 3 filter paper in November-December 2006 (baseline), 2007, and 2008. Dried filter papers were kept at $-20^{\circ} \mathrm{C}$ until the direct agglutination test was performed as described elsewhere. ${ }^{22}$ A training workshop was organised to standardise the test protocol. Filter papers were analysed by survey batches to minimise processing errors, and $10 \%$ of the filter papers per survey were re-tested for quality control. No systematic errors were detected, and agreement between laboratories was good.

We assessed the number of incident cases of visceral leishmaniasis as a secondary outcome during quarterly house to house surveys from November 2006 to May 2009. Information about any case in the family was double checked with patients' records. People with fever lasting for two weeks or more were examined by a physician and tested with a rapid diagnostic test for visceral leishmaniasis (Kalazar Detect Rapid Test; InBios International, Seattle, WA).$^{23}$ All clinically suspected cases detected during the trial were classified as probable or certain visceral leishmaniasis by a clinician

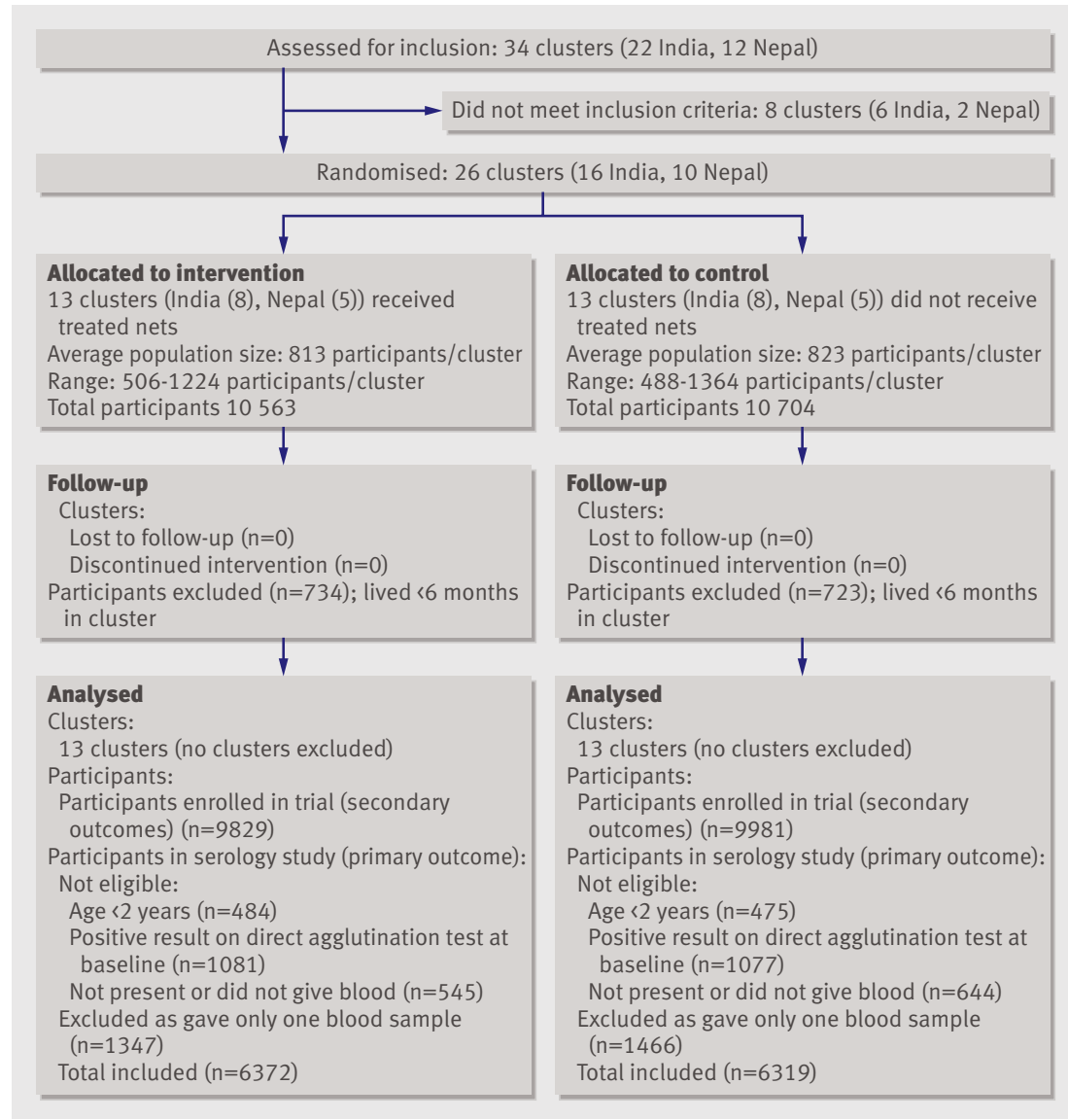

Fig 1 | Trial profile of clusters and individuals allocated to use of longlasting insecticidal nets or control who was blinded to the status of the cluster. Suspected cases not corresponding to case definitions (see appendix on bmj.com) or with a positive direct agglutination test result at baseline were excluded. We excluded from the analysis cases of visceral leishmaniasis with onset of disease within two months after the baseline or more than two months after the final survey (to take into account the minimum incubation period) ${ }^{24}$. Asymptomatic infections were clinically followed up for a minimum of six months. Malaria was diagnosed during quarterly house to house surveys with a rapid diagnostic test (Parascreen; Zephyr Biomedicals, Goa, India $)^{25}$ in people with fever. Trained field workers carried out verbal autopsies on all deaths recorded during the trial. Two independent physicians ascertained cause of death.

\section{Statistical methods}

All data were double entered into an EpiInfo 2000 database (Center for Disease Control and Prevention, Atlanta, GA) and analysed in Stata 11 (StataCorp LP, College Station, TX).

The data were analysed at cluster level, and the overall effect of the intervention was expressed as a risk ratio (treated nets/no treated nets) for $L$ donovan $i$ infection. This overall estimate was obtained by taking the geometric mean of the risk ratios for seroconversion of each matched cluster pair. Formal tests of no intervention effect and confidence intervals were obtained with an unweighted paired $t$ test on the log scale. ${ }^{26}$ The confidence interval was computed on the log scale and then back transformed to the original scale by taking the anti-log.

Adjusted analyses were carried out in two stages. Firstly, we used a standard individual level logistic regression model to calculate expected number of events for each cluster ignoring the intervention. This standard model included pair and was simultaneously adjusted for age group, sex, indoor residual spraying, and socioeconomic status. Observed and expected values were used to calculate residuals as the ratio of observed and expected. The adjusted intervention effect was calculated with these residuals in a paired $t$ test.

For secondary outcomes (such as visceral leishmaniasis and malaria) and some subgroup analyses (such as $L$ donovani infection by socioeconomic status group) where the outcomes were rare and a cluster was left without an event, we calculated the risk ratio as the ratio of the arithmetic mean of proportions in intervention and control arms using the variance suggested by Hayes and Moulton ${ }^{26}$ to obtain approximate $95 \%$ confidence intervals and test probabilities, thus ignoring the pairing in these situations.

For the adjusted analyses, we calculated a composite index reflecting the socioeconomic status of each household using principal components analysis to aggregate wealth, housing, and demographic characteristics. ${ }^{327}$ Five equally sized groups were created for household socioeconomic status and age at baseline. The number of times each household was sprayed during the trial was grouped as none, once, 
Table 1|Baseline (November-December 2006) demographic information in intervention (longlasting insecticidal nets) and control groups for total study population. Figures are percentages (numbers) unless stated otherwise

\begin{tabular}{|c|c|c|}
\hline Variable & Intervention & Control \\
\hline \multicolumn{3}{|l|}{ Total } \\
\hline No of participants & 9829 (13 clusters) & 9981 (13 clusters) \\
\hline Mean (SD) age (years) & $22.7(19.2)$ & $23.0(19.2)$ \\
\hline No (\%) male & $4858(49.4)$ & $4916(49.2)$ \\
\hline Annual incidence of visceral leishmaniasis 2003-5 & $1.4(418 / 9260)$ & $1.5(410 / 8722)$ \\
\hline Positive direct agglutination test results at baseline & $14.9(1039 / 6975)$ & $14.5(940 / 6473)$ \\
\hline No (range) of households/cluster & 1690 (78 to 240$)$ & 1803 (88 to 224) \\
\hline Mean (SD) No of members in household & $5.8(2.7)$ & $5.5(2.5)$ \\
\hline Clusters sprayed before intervention* & 6 & 6 \\
\hline Households with untreated nets $(95 \% \mathrm{Cl}) \dagger$ & $76.2(67.2$ to 85.1$)$ & $78.1(69.3$ to 87.0$)$ \\
\hline Mean indicator of socioeconomic statusł (SD) & $1.9(1.4)$ & $2.1(1.4)$ \\
\hline \multicolumn{3}{|l|}{ India } \\
\hline No of participants & 5987 (8 clusters) & 6207 (8 clusters) \\
\hline Mean (SD) age (years) & $21.9(19.7)$ & $22.4(19.6)$ \\
\hline No (\%) male & $2980(49.8)$ & $3067(49.4)$ \\
\hline Annual incidence of visceral leishmaniasis $2003-5 \pi$ & $1.51(279 / 5677)$ & $1.40(261 / 5640)$ \\
\hline Positive direct agglutination test results at baseline & $19.4(785 / 4059)$ & $17.7(705 / 3992)$ \\
\hline No (range) of households/cluster & 967 (78 to 172$)$ & 1082 (88 to 224) \\
\hline Mean (SD) No of members in household & $6.2(3.0)$ & $5.7(2.7)$ \\
\hline Clusters sprayed before intervention* & 1 & 3 \\
\hline Households with untreated nets $(95 \% \mathrm{Cl}) \dagger$ & $81.4(70.5$ to 92.3$)$ & $81.5(67.0$ to 95.9$)$ \\
\hline Mean indicator of socioeconomic statusł (SD) & $2.0(1.4)$ & $2.0(1.5)$ \\
\hline \multicolumn{3}{|l|}{ Nepal } \\
\hline No of participants & 3842 (5 clusters) & 3774 (5 clusters) \\
\hline Mean (SD) age (years) & $23.9(18.6)$ & $23.9(18.3)$ \\
\hline No (\%) male & $1878(48.9)$ & $1849(49.0)$ \\
\hline Annual incidence of visceral leishmaniasis 2003-5 & $1.3(139 / 3583)$ & $1.6(149 / 3082)$ \\
\hline Positive direct agglutination test results at baseline & $8.7(254 / 2916)$ & $9.5(235 / 2481)$ \\
\hline No (range) of households/cluster & 723 (103 to 240$)$ & 721 (103 to 196) \\
\hline Mean (SD) No of members in household & $5.3(2.2)$ & $5.2(2.3)$ \\
\hline Clusters sprayed before intervention* & 5 & 3 \\
\hline Households with untreated nets $(95 \% \mathrm{Cl}) \dagger$ & $67.8(49.6$ to 86.0$)$ & $72.8(62.7$ to 82.9$)$ \\
\hline Mean indicator of socioeconomic statusł (SD) & $1.9(1.4)$ & $2.1(1.4)$ \\
\hline
\end{tabular}

*July 2005 to November 2006.

†At least one untreated net observed by study teams. Percentage estimated from survey in 25 households per cluster conducted in August-September 2006. ${ }^{13}$

$\ddagger$ Composite index calculated for each household with principal components analysis to aggregate wealth, housing, and demographic characteristics. ${ }^{27}$ Household indexes categorised in five equally sized groups.

IIncidence in nine clusters (four in intervention) calculated from 3.5 years.

and twice or more. Similarly, we tested whether the intervention effect was the same in India and Nepal.

\section{Ethical considerations}

Communities were informed about the purpose of the trial and consent for inclusion was sought from village leaders. Written informed consent was obtained from head of households and from each individual or their guardian for those aged under 18. For ethical reasons, we conducted an interim analysis 12 months after the intervention, with a pre-planned rule to stop the trial and distribute treated nets in the control clusters if the $95 \%$ confidence interval indicated a $30 \%$ or greater reduction in the incidence rate of visceral leishmaniasis in the intervention group compared with the control group.

\section{RESULTS}

Participants flow

Out of 15504 eligible seronegative people, we included $12691(82 \%)$ in the analysis of the main outcome (infection) and considered 19810 of the 21267 $(93 \%)$ initially enrolled for the secondary (disease) end point. Figure 1 shows reasons for exclusion and the range and number of households and people per cluster and allocation group. The proportion of people lost to follow-up (not present or with one or no blood sample) was slightly higher in the control group $(21 \%,(644$ $+1466) / 9981)$ than in the intervention group $(19 \%$ $(545+1347) / 9829)$. But the characteristics of the participants lost to follow-up in both groups were similar (mean age $22 v 23$, males 62\% v63\%, mean socioeconomic status $2.0 \vee 2.2$, in intervention and control groups respectively). Intervention and control groups were well balanced at individual and cluster levels, but the prevalence of positive results on the direct agglutination test at baseline in India was almost twice as high as in Nepal, despite the previous annual incidence of visceral leishmaniasis being similar (table 1). Participants in the group in which the infection end point was measured - that is, those with negative results on direct agglutination test at baseline-were slightly older and more were female than the general population, but, again, this was well balanced between study groups (table 2). Vector densities at baseline were comparable in intervention and control clusters. ${ }^{11}$ During the two year follow-up, 14 clusters (six intervention and eight control) were sprayed under the national control programme with limited or no effect on vector density. ${ }^{11}$ In intervention clusters, $8920 / 9829(91 \%)$ of the individuals slept regularly (that is, over $80 \%$ of the nights) under a treated net. Those observations were confirmed by an additional acceptability survey (V Vanlerberghe, personal communication, January 2010). The use of untreated nets in the control group was variable; 7012/9981 (70\%) used a bed net at least once during the trial but only 2978/9981 (30\%) used it regularly throughout the year as most of the households did not have enough nets for all their members.

\section{Outcomes and estimation}

Primary outcome

The number of individuals who seroconverted ranged from 5 to 60 per cluster, and the incidence of infection was variable among cluster pairs (fig 2). The risk of seroconversion during the two year follow-up was significantly different between countries: $7.2 \%$ in India $(529 / 7368)$ and $3.1 \%(163 / 5323)$ in Nepal. The overall risk of seroconversion in the intervention $(5.4 \%, 347 /$ $6372)$ and control $(5.5 \%, 345 / 6319)$ groups was similar. At cluster level, the risk of infection was reduced by $10 \%$ in the intervention clusters compared with contro clusters, but this effect was not significant (risk ratio $0.90,95 \%$ confidence interval 0.49 to 1.65 ). The results were similar in the model adjusted for age, sex, indoor residual spraying, and socioeconomic status $(0.89,0.48$ to 1.64) (table 3). Longlasting insecticidal nets seemed to have an opposite effect on seroconversion according 
Table 2 Baseline demographic information in intervention (longlasting insecticidal nets) and control groups for eligible subgroups observed for main outcome (seroconversion on direct agglutination test)

\begin{tabular}{lcc} 
Variable & Intervention & Control \\
Total & & \\
\hline No of participants & $6372(13$ clusters) & 6319 (13 clusters) \\
\hline Mean (SD) age (years) & $23.6(19.1)$ & $23.3(19.0)$ \\
\hline No (\%) male & $2882(45.2)$ & $2809(44.5)$ \\
\hline India & & \\
\hline No of participants & $3568(8$ clusters) & 3800 (8 clusters) \\
\hline Mean (SD) age (years) & 22.9 (19.8) & $22.4(19.3)$ \\
\hline No (\%) male & 1570 (44.0) & $1669(43.9)$ \\
\hline Nepal & & \\
\hline No of participants & $2804(5$ clusters) & $2519(5$ clusters) \\
\hline Mean (SD) age (years) & $24.5(18.3)$ & $24.7(18.4)$ \\
\hline No (\%) male & 1312 (46.8) & $1140(45.3)$ \\
\hline
\end{tabular}

to the direct agglutination test result in India $(1.09,0.58$ to 2.04$)$ and Nepal $(0.66,0.12$ to 3.56$)$, but the interaction was not significant $(\mathrm{P}=0.40)$. Similar results were obtained in the adjusted model: risk ratio 1.09 and 0.57 in India and Nepal, respectively (table 3).

\section{Secondary outcomes}

A total of 168 suspected cases of visceral leishmaniasis (129 in India and 39 in Nepal) were identified during the trial. The case ascertainment procedure reduced this number to 77 confirmed cases; 62 in India and 15 in Nepal (fig 3). The overall risk of visceral leishmaniasis during the two year follow-up was $0.38 \%$ (37/9829) and $0.40 \%(40 / 9981)$ in the intervention group and control group, respectively. The cluster level analysis showed that longlasting insecticidal nets reduced the risk of visceral leishmaniasis by $1 \%$ (risk ratio 0.99 , $95 \%$ confidence interval 0.46 to 1.40 ), but the effect was not significant. The country specific and adjusted results were similar and not significant (table 3 ). We obtained analogous results when we analysed all suspected cases and when we used a six month incubation period (see appendix on bmj.com).

A total of 225 cases of malaria were identified, mostly in India $(\mathrm{n}=220)$ and mostly non- $P$ falciparum malaria $(\mathrm{n}=208)$; there were 12 cases of $P$ falciparum malaria and five undefined species. There was a nonsignificant reduction in cases of malaria in intervention clusters (risk ratio $0.63,0.29$ to 1.36 ), but the adjusted effect was stronger and significant $(0.46,0.28$ to 0.77$)$. In India, where most malaria cases were reported, the risk ratio was 0.60 (0.38 to 0.94 ) in the adjusted model (table 3). Similarly, the total number of deaths was reduced in intervention clusters $(0.75,0.50$ to 1.13$)$, but the effect was not significant (table 3). None of the deaths during the trial was attributable to visceral leishmaniasis.

\section{DISCUSSION}

In this large scale randomised controlled trial of the effectiveness of longlasting insecticidal nets in preventing visceral leishmaniasis, we found the risk for $L$ donovani infection was reduced by $10 \%$ in intervention clusters. This was a small and non-significant effect, though its wide confidence interval does not rule out a potential beneficial effect. The findings in the primary (serological) and secondary (clinical visceral leishmaniasis) end points were consistent as there was no difference in the incidence of visceral leishmaniasis between intervention and control clusters. Use of the treated nets in intervention clusters was high in all seasons and significantly reduced the risk of malaria, especially in India, where $98 \%$ of all malaria cases in this trial were reported.

\section{Strengths and limitations}

The incidence of Leishmania infection in study clusters could have been lowered by the active detection and treatment of cases of visceral leishmaniasis implemented during the trial but did not fall below the assumption of $2 \%$ annual incidence made when we calculated the sample size. If we assume a constant intervention effect over all pairs, the observed coefficient of variation for $L$ donovani infection was 0.56 . This value can be of use for the planning of future intervention trials. While our sample size calculation was based on a lower $\kappa$ than the one finally observed, the power of the study was increased by adding six extra clusters. The impact of migration on the estimation of effect was limited as the number of individuals excluded was similar in both groups. Follow-up rates during the trial were high, with $82 \%$ and $93 \%$ for the primary and secondary outcome, respectively. The proportion lost to follow-up was slightly higher in control (21\%) than in intervention (19\%) clusters, but we do not expect this to affect the estimations as the characteristics of these people were similar. We used a cluster level model to analyse the trial data as this was the analytical model specified for the sample size formula ${ }^{17}$ and is the method recommended by Hayes and Moulton $^{26}$ for pair matched trials with fewer than 15 clusters per arm. The trial results were not significantly modified when we used a multi-level model (random effects logistic regression) (see appendix on bmj.com).

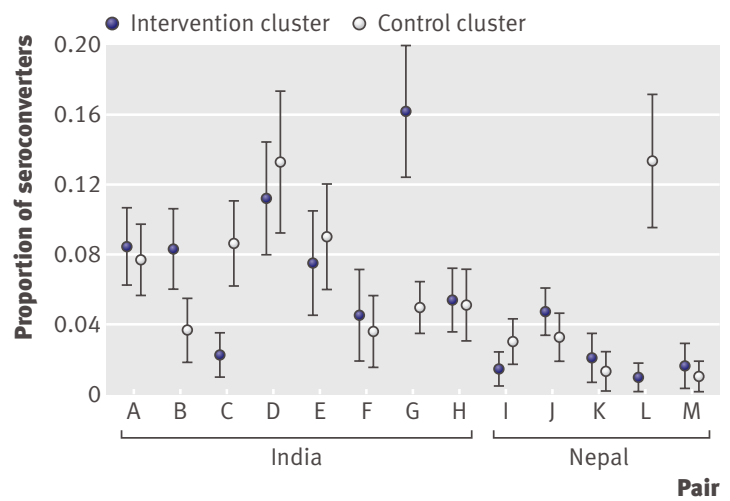

Fig 2 | Observed proportion of seroconversions according to result of direct agglutination test by cluster and pair with $95 \%$ confidence intervals 
Table 3 |Effect of longlasting insecticidal nets on $L$ donovani infection measured by direct agglutination test with cut off 1:1600 and minimum of two titres difference and secondary outcomes: visceral leishmaniasis, malaria cases and all causes of death. Overall and country specific unadjusted and adjusted risk ratios for intervention compared with control from cluster analysis

\begin{tabular}{|c|c|c|c|c|}
\hline \multirow[b]{2}{*}{ Variable } & \multirow[b]{2}{*}{ Intervention } & \multirow[b]{2}{*}{ Control } & \multicolumn{2}{|c|}{ Risk ratio $(95 \% \mathrm{Cl})$, P value* } \\
\hline & & & Unadjusted & Adjusted $†$ \\
\hline \multicolumn{5}{|l|}{ Overall } \\
\hline No in serology study & 6372 (13 clusters) & 6319 (13 clusters) & - & - \\
\hline No of seroconversions (\%) & $347(5.4)$ & $345(5.5)$ & 0.90 (0.49 to 1.65$), 0.71$ & 0.89 (0.48 to 1.64$), 0.68$ \\
\hline Total No of participants & 9829 (13 clusters) & 9981 (13 clusters) & - & - \\
\hline Visceral leishmaniasis (\%)‡ & $37(0.38)$ & $40(0.40)$ & 0.99 (0.46 to 2.16), 0.99 & 1.15 (0.61 to 2.16$), 0.64$ \\
\hline Malaria (\%) $\ddagger$ & $88(0.90)$ & $137(1.37)$ & 0.63 (0.29 to 1.36$), 0.21$ & 0.46 (0.28 to 0.77$), 0.01$ \\
\hline All causes of death (\%) & $124(1.26)$ & $167(1.67)$ & 0.75 ( 0.50 to 1.13$), 0.15$ & 0.78 (0.56 to 1.10$), 0.15$ \\
\hline \multicolumn{5}{|l|}{ India } \\
\hline No in serology study & 3568 (8 clusters) & 3800 (8 clusters) & - & - \\
\hline No of seroconversions (\%) & $276(7.7)$ & $253(6.7)$ & 1.09 (0.58 to 2.04), 0.76 & 1.09 (0.58 to 2.05$), 0.75$ \\
\hline Total No of participants & 5988 (8 clusters) & 6207 (8 clusters) & - & - \\
\hline Visceral leishmaniasis (\%) $\ddagger$ & $31(0.52)$ & $31(0.50)$ & 1.00 (0.41 to 2.44$), 1.00$ & 0.94 (0.44 to 2.02), 0.85 \\
\hline Malaria (\%) $\ddagger$ & $87(1.45)$ & $133(2.14)$ & 0.64 (0.36 to 1.13$), 0.11$ & 0.60 (0.38 to 0.94$), 0.03$ \\
\hline All causes of death (\%) & $80(1.34)$ & $121(1.95)$ & 0.62 (0.32 to 1.19), 0.13 & 0.72 (0.44 to 1.19$), 0.17$ \\
\hline \multicolumn{5}{|l|}{ Nepal } \\
\hline No in serology study & 2804 (5 clusters) & 2519 (5 clusters) & - & - \\
\hline No of seroconversions (\%) & $71(2.5)$ & $92(3.6)$ & $0.66(0.12$ to 3.56$), 0.53$ & 0.57 (0.11 to 2.97$), 0.40$ \\
\hline Total No of participants & 3842 (5 clusters) & 3774 (5 clusters) & - & - \\
\hline Visceral leishmaniasis (\%)‡ & $6(0.16)$ & $9(0.24)$ & 0.96 (0.13 to 7.39$), 0.96$ & 1.55 (0.17 to 14.18$), 0.57$ \\
\hline Malaria (\%) & $1(0.03)$ & $4(0.11)$ & 0.18 (0.00 to 14.38$), 0.23$ & -§ \\
\hline All causes of death (\%) & $44(1.15)$ & $46(1.22)$ & 1.02 (0.67 to 1.55$), 0.93$ & 1.06 (0.69 to 1.64$), 0.73$ \\
\hline
\end{tabular}

*Test for no intervention effect.

†Adjusted for age group, sex, times sprayed, and socioeconomic status.

$\ddagger$ Rare outcomes: some clusters did not record any event during study period. Risk ratio calculated as ratio of arithmetic mean of proportions in intervention and control arm with variance (see appendix on bmj.com).

$\S$ Too few cases of malaria to adjust for covariates.

We used the direct agglutination test as a marker for infection, as in previous epidemiological studies, ${ }^{16}{ }^{1928-30}$ because the low incidence of visceral leishmaniasis precluded its use as primary outcome. We found no published estimates on the sensitivity and specificity of the direct agglutination test to test for infection in humans, but we have shown that recent seroconverters are at a substantially higher risk of developing visceral leishmaniasis in this setting. ${ }^{31}$ The trial outcome was not modified when less (that is, seroconversion based on a 1 titre difference) or more (that is, higher direct agglutination test cut offs) specific criteria were used (see appendix on bmj.com). Though others have used the leishmanin skin test as a marker of exposure, we could not do so in our trial because of unsatisfactory performance in India. ${ }^{32}$

\section{Interpretation}

As adherence to use of the treated nets was high during the trial, the lack of effect could be explained by biological factors. World Health Organization pesticide evaluation scheme (WHOPES) standard susceptibility tests showed that $P$ argentipes is susceptible to deltamethrin in the study clusters. ${ }^{33}$ Moreover, indoor density of $P$ argentipes was reduced by $25 \%$ in the study clusters that used treated nets compared with control clusters. ${ }^{11}$ This reduction, however, might be insufficient to have an impact on immunological and clinical outcomes if, in contrast with previous data, ${ }^{5}$ transmission of $L$ donovani occurs partly outside the house, as suggested by recent studies. ${ }^{9345}$ This trial measured the effectiveness of distribution of treated nets in a real life context, where some clusters were sprayed and some people in control clusters used untreated nets. The use of untreated nets before the trial did not prevent all transmission in these communities. Analysis showed that protection provided by untreated nets was similar to that from treated nets; whereas both treated and untreated nets seemed to provide some degree of protection compared with not using nets (adjusted models $\mathrm{P}<0.06$ and $\mathrm{P}<0.09$, respectively) (see appendix on bmj.com). This is supported by entomological data from the study clusters. ${ }^{36}$

The fact that 14 of the 26 study clusters were sprayed as part of the visceral leishmaniasis national control programmes during the trial had a limited impact on vector density, as reported elsewhere. ${ }^{11}$ Moreover, the effect of treated nets on $L$ donovani infection was not significantly modified in the adjusted models, which included indoor residual spraying as a variable. Under controlled conditions, indoor residual spraying effectively reduces the indoor density of $P$ argentipes. ${ }^{10}$ When applied as part of the national vector control programmes, however, indoor residual spraying was 
Cases of suspected visceral leishmaniasis, November 2006 to May 2009 (total=168; India=129, Nepal=39)

\begin{tabular}{|c|c|}
\hline Treated nets (total=76; India=55, Nepal=21) & Control group (total=92; India $=74, \mathrm{Nepal}=18$ ) \\
\hline$\downarrow$ & $\downarrow$ \\
\hline $\begin{array}{l}\text { Excluded }(n=39) \text { : } \\
\text { Lived }<6 \text { months in cluster }\end{array}$ & Excluded $(n=52)$ : \\
\hline (total=7; India=6, Nepal=1) & $\begin{array}{l}\text { Lived }<6 \text { months in cluster } \\
\text { (total }=5 ; \text { India }=5, \text { Nepal }=0 \text { ) }\end{array}$ \\
\hline $\begin{array}{l}\text { Post-kala-azar dermal leishmaniasis } \\
\text { (total }=1 ; \text { India }=1, \text { Nepal }=0 \text { ) }\end{array}$ & $\begin{array}{l}\text { Post-kala-azar dermal leishmaniasis } \\
\text { (total }=3 ; \text { India }=3 \text {, Nepal=0) }\end{array}$ \\
\hline $\begin{array}{l}\text { Possible visceral leishmaniasis } \\
\text { (total=1, India }=1, \text { Nepal=0) }\end{array}$ & $\begin{array}{l}\text { Possible visceral leishmaniasis } \\
\text { (total=4, India=4, Nepal=0) }\end{array}$ \\
\hline $\begin{array}{l}\text { Positive result on direct agglutination test at } \\
\text { baseline (total=12; India }=6 \text {, Nepal=6) }\end{array}$ & $\begin{array}{l}\text { Positive result on direct agglutination test at } \\
\text { baseline (total=8; India=7, Nepal=1) }\end{array}$ \\
\hline Relapse (total=1, India $=0$, Nepal=1) & Relapse (total=3, India $=2$, Nepal $=1)$ \\
\hline $\begin{array}{l}\text { Symptoms started }<2 \text { months after intervention } \\
\text { (total=6, India }=3 \text {, Nepal=3) }\end{array}$ & $\begin{array}{l}\text { Symptoms started }<2 \text { months after intervention } \\
\text { (total }=10, \text { India }=7, \text { Nepal }=3 \text { ) }\end{array}$ \\
\hline $\begin{array}{l}\text { Symptoms started }>2 \text { months after completion } \\
\text { of trial (total }=3 \text {, India }=3 \text {, Nepal }=0 \text { ) }\end{array}$ & $\begin{array}{l}\text { Symptoms started }>2 \text { months after completion } \\
\text { of trial (total }=1 \text {, India }=1, \text { Nepal }=0 \text { ) }\end{array}$ \\
\hline $\begin{array}{l}\text { Combination of reasons for exclusion } \\
\text { (total }=8, \text { India }=4, \mathrm{Nepal}=4 \text { ) }\end{array}$ & $\begin{array}{l}\text { Combination of reasons for exclusion } \\
\text { (total }=18, \text { India }=14, \text { Nepal }=4 \text { ) }\end{array}$ \\
\hline$\downarrow$ & $\downarrow$ \\
\hline Analysed (total=37; India=31, Nepal=6) & Analysed (total=40; India=31, Nepal=9) \\
\hline
\end{tabular}

Fig 3 | Case ascertainment for visceral leishmaniasis and reasons for exclusion

shown to have a limited effect or no effect on indoor density of $P$ argentipes in villages in Bihar with endemic visceral leishmaniasis ${ }^{9}$ and, more generally, on control of visceral leishmaniasis. ${ }^{6}$ The poor quality of routine indoor residual spraying operations for visceral leishmaniasis in the region is the main reason for this failure $^{37}$ and has contributed to the development of DDT resistance in districts with endemic visceral leishmaniasis ${ }^{6}$ and in our study areas in particular. ${ }^{33}$ Similarly, the variability in incidence observed in some study clusters-notably, pairs G and L (see fig 2)-does not seem to be explained by the effect of indoor residual spraying as both clusters in pair $\mathrm{G}$ were sprayed once and neither cluster was sprayed in pair L. The differences could be better explained by heterogeneity in the transmission dynamics (because of, for example, heterogeneity in population susceptibility and vector infectivity). This variability does not seem to affect the trial outcomes, as the effect of treated nets on $L$ donovani infection was not significantly altered when we repeated the analyses excluding the clusters with exceptionally high rates (outliers) (see appendix on bmj.com).

\section{WHAT IS ALREADY KNOWN ON THIS TOPIC}

Longlasting insecticidal nets are among the major breakthroughs in the control of malaria in recent years, and expectations were raised about their potential to prevent other vectorborne diseases

Observational evidence in East Africa and Asia suggested that people sleeping under nets were protected against visceral leishmaniasis

\section{WHAT THIS STUDY ADDS}

Village-wide distribution of insecticide treated nets in India and Nepal did not confer protection against Leishmania donovani infection or visceral leishmaniasis when compared with existing preventive practice (irregular insecticide residual spraying and use of untreated nets)
Our results are in contrast with those of an observational study in Sudan, which reported a 27\% reduction in cases with a smaller mesh size. ${ }^{12}$ The mesh size used in this trial $(2 \mathrm{~mm})$ would not be a perfect physical barrier for sandflies, but the insecticide coating of the fibres should prevent their passage. ${ }^{38}$

\section{Implications}

Our results show that the distribution of longlasting insecticidal nets did not confer additional protection against $L$ donovani infection and visceral leishmaniasis in the current context in endemic communities in India and Nepal when compared with existing control practices (that is, irregular indoor residual spraying, use of untreated nets, and treatment of visceral leishmaniasis). Also distribution of treated nets did not reduce the annual incidence of visceral leishmaniasis below 18.8 per 10000 , which by far exceeds the elimination target in the region. ${ }^{7}$ The most biologically plausible explanation for our results is that a substantial fraction of $L$ donovani transmission occurs outside the house, where any nets would have less impact on preventing sandfly-human contact. Nonetheless, insecticide treated nets should not be dismissed as a potential intervention for visceral leishmaniasis prevention on the Indian subcontinent, as more research on vector behaviour and implementation of such nets is warranted.

The KALANET community trial was part of a large project conducted by the KALANET consortium funded by the European Union under its $6^{\text {th }}$ Framework Program (INCODEV/Project 015374). We are grateful to the co-investigators in the KALANET consortium who conducted the socioeconomic, biological, or technological studies that generated extremely valuable information for interpretation of the trial data. We thank the members of the scientific advisory board (C Lengeler (chair), A Kroeger, M Maroli, C Prasittisuk, J Alvar, B Mahendra, and S Bhattacharya) for their comments; A Huys and C Albrecht for administrative support; I Menten for statistical support; $F$ Meheus for computation of the socioeconomic index; V Lejon, D Jacquet, I Wouters, and I Swiers for the quality control of the laboratory activities; B Greenwood and P Milligan for their comments on the manuscript; $V$ K Dubey, B Pathak, and all field workers of the KALANET consortium and the population of the villages who took part in the trial. VestergaardFrandsen provided free PermaNets for distribution in control clusters at the end of the trial. Dedicated to the memory of Clive Richard Davies, who died in March 2009.

Contributors: AP, SPS, SR, SS, FC, VV, CRD, and MB designed the trial. AP, SPS, BO, FC, SU, KG, RK, PS, BK, ISP, and MLD collected data. AP, SPS, SR, $\mathrm{SS}, \mathrm{BO}, \mathrm{FC}, \mathrm{JCD}, \mathrm{MR}, \mathrm{VV}, \mathrm{EWA}, \mathrm{CRD}$, and MB analysed and interpreted data. All authors wrote the paper. MB is guarantor.

Funding: This study was funded by the European Union under its 6th Framework Program (INCODEV/Project 015374). The funder of the study had no role in the study design, data collection and analysis,

interpretation or reporting of this work, or the decision to submit the work for publication. European Commission: Contract no INCO-CT 200501537, KALANET project.

Competing interests: All authors have completed the Unified Competing Interest form at www.icmje.org/coi_disclosure.pdf (available on request from the corresponding author) and declare: no support from any organisation for the submitted work; no financial relationships with any organisations that might have an interest in the submitted work in the previous three years, no other relationships or activities that could appear to have influenced the submitted work.

Ethical approval: Communities were informed about the purpose of the trial and consent for inclusion was sought from village leaders. Written informed consent was obtained from head of households and from each individual or their guardian for those aged under 18. Clearance was obtained from the ethical committees of Institute of Medical Sciences (Banaras Hindu University), B P Koirala Institute of Health Sciences, the 
London School of Hygiene and Tropical Medicine, and the University of Antwerp.

Data sharing: Technical appendix, statistical code, and dataset available from the corresponding author at mboelaert@itg.be. Participants' consent for data sharing was not obtained but the presented data are anonymised and risk of identification is low.

1 Hotez PJ, Molyneux DH, Fenwick A, Kumaresan J, Sachs SE, Sachs JD, et al. Control of neglected tropical diseases. N Engl J Med 2007;357:1018-27.

2 Desjeux P. Leishmaniasis: current situation and new perspectives. Comp Immunol Microbiol Infect Dis 2004;27:305-18.

3 Boelaert M, Meheus F, Sanchez A, Singh SP, Vanlerberghe V, Picado A, et al. The poorest of the poor: a poverty appraisal of households affected by visceral leishmaniasis in Bihar, India. Trop Med Int Health 2009;14:639-44.

4 Swaminath CS, Shortt HE, Anderson LA. Transmission of Indian kalaazar to man by the bites of Phlebotomus argentipes, ann and brun. 1942. Indian J Med Res 2006;123:473-7.

5 Dinesh DS, Ranjan A, Palit A, Kishore K, Kar SK. Seasonal and nocturnal landing/biting behaviour of Phlebotomus argentipes (Diptera: Psychodidae). Ann Trop Med Parasitol 2001;95:197-202.

6 Ostyn B, Vanlerberghe V, Picado A, Dinesh DS, Sundar S, Chappuis F, et al. Vector control by insecticide-treated nets in the fight against visceral leishmaniasis in the Indian subcontinent, what is the evidence? Trop Med Int Health 2008;13:1073-85.

7 Bhattacharya SK, Sur D, Sinha PK, Karbwang I. Elimination of leishmaniasis (kala-azar) from the Indian subcontinent is technically feasible and operationally achievable. Indian J Med Res 2006;123:195-6.

8 Lengeler $C$. Insecticide-treated bed nets and curtains for preventing malaria. Cochrane Database Syst Rev 2004;2:CD000363.

9 Dinesh DS, Das P, Picado A, Davies C, Speybroeck N, Ostyn B, et al. Long-lasting insecticidal nets fail at household level to reduce abundance of sandfly vector Phlebotomus argentipes in treated houses in Bihar (India). Trop Med Int Health 2008;13:953-8.

10 Joshi AB, Das ML, Akhter S, Chowdhury R, Mondal D, Kumar V, et al. Chemical and environmental vector control as a contribution to the elimination of visceral leishmaniasis on the Indian subcontinent: cluster randomized controlled trials in Bangladesh, India and Nepal. BMC Med 2009;7:54

11 Picado A, Das ML, Kumar V, Kesari S, Dinesh DS, Roy L, et al. Effect of village-wide use of long-lasting insecticidal nets on visceral leishmaniasis vectors in India and Nepal: a cluster randomized trial. PLoS Negl Trop Dis 2010;4:e587.

12 Ritmeijer K, Davies C, van Zorge R, Wang SJ, Schorscher J, Dongu'du $\mathrm{SI}$, et al. Evaluation of a mass distribution programme for fine-mesh impregnated bednets against visceral leishmaniasis in eastern Sudan. Trop Med Int Health 2007;12:404-14.

13 Vanlerberghe V, Singh SP, Paudel IS, Ostyn B, Picado A, Sanchez A, et al. Determinants of bednet ownership and use in visceral leishmaniasis-endemic areas of the Indian subcontinent. Trop Med Int Health 2010;15:60-7.

14 Bern C, Hightower AW, Chowdhury R, Ali M, Amann J, Wagatsuma Y, et al. Risk factors for kala-azar in Bangladesh. Emerg Infect Dis 2005;11:655-62.

15 Bern C, Joshi AB, Jha SN, Das ML, Hightower A, Thakur GD, et al. Factors associated with visceral leishmaniasis in Nepal: bed-net use is strongly protective. Am J Trop Med Hyg 2000;63:184-8.

16 Saha S, Ramachandran R, Hutin YJF, Gupte MD. Visceral leishmaniasis is preventable in a highly endemic village in West Bengal, India. Trans R Soc Trop Med Hyg 2009;103:737-42.

17 Hayes RJ, Bennett S. Simple sample size calculation for clusterrandomized trials. Int J Epidemiol 1999;28:319-26.

18 Rijal S, Uranw S, Chappuis F, Picado A, Khanal B, Paudel IS, et al. Epidemiology of Leishmania donovani infection in high-transmission foci in Nepal. Trop Med Int Health 2010;15:21-8.
19 Davies CR, Mazloumi Gavgani AS. Age, acquired immunity and the risk of visceral leishmaniasis: a prospective study in Iran. Parasitology 1999;119:247-57.

20 Chappuis F, Rijal S, Soto A, Menten J, Boelaert M. A meta-analysis of the diagnostic performance of the direct agglutination test and rK39 dipstick for visceral leishmaniasis. BMJ 2006;333:723.

21 Boelaert M, El Safi S, Mousa H, Githure J, Mbati P, Gurubacharya V, et al. Multi-centre evaluation of repeatability and reproducibility of the direct agglutination test for visceral leishmaniasis. Trop Med Int Health 1999:4:31-7.

22 Jacquet D, Boelaert M, Seaman J, Rijal S, Sundar S, Menten J, et al. Comparative evaluation of freeze-dried and liquid antigens in the direct agglutination test for serodiagnosis of visceral leishmaniasis (ITMA-DAT/VL). Trop Med Int Health 2006;11:1777-84.

23 Sundar S, Maurya R, Singh RK, Bharti K, Chakravarty J, Parekh A, et al. Rapid, noninvasive diagnosis of visceral leishmaniasis in India: comparison of two immunochromatographic strip tests for detection of anti-K39 antibody. J Clin Microbiol 2006;44:251-3.

24 Chappuis F, Sundar S, Hailu A, Ghalib H, Rijal S, Peeling RW, et al. Visceral leishmaniasis: what are the needs for diagnosis, treatment and control? Nat Rev Microbiol 2007;5:873-82.

25 World Health Organization. Malaria rapid diagnostic test performance-results of WHO product testing of malaria RDTs: round 1 (2008). TDR/WHO, 2009.

26 Hayes RJ, Moulton LH. Cluster randomised trials. Chapman and Hall/ CRC, 2009.

27 Singh SP, Picado A, Boelaert M, Gidwani K, Andersen EW, Ostyn B, et al. The epidemiology of Leishmania donovani infection in high transmission foci in India. Trop Med Int Health 2010;15:12-20.

28 Chowdhury MS, el Harith A, al Massum A, al Karim E, al Rahman A. Prevalence of agglutinating anti-Leishmania antibodies in two multithousand Bengoli communities. Parasitol Res 1993;79:444-50.

29 Koirala S, Karki P, Das ML, Parija SC, Karki BM. Epidemiological study of kala-azar by direct agglutination test in two rural communities of eastern Nepal. Trop Med Int Health 2004;9:533-7.

30 Schenkel K, Rijal S, Koirala S, Koirala S, Vanlerberghe V, Van der Stuyft P, et al. Visceral leishmaniasis in southeastern Nepal: a crosssectional survey on Leishmania donovani infection and its risk factors. Trop Med Int Health 2006;11:1792-9.

31 Ostyn B, Gidwani K, Picado A, Chappuis F, Khanal B, Singh S, et al. Incidence of asymptomatic infection with $L$ donovani and their evolution in high endemic villages in India and Nepal. Trop Med Int Health 2009;14:60-1.

32 Gidwani K, Rai M, Chakravarty J, Boelaert M, Sundar S. Evaluation of leishmanin skin test in Indian visceral leishmaniasis. Am J Trop Med Hyg 2009;80:566-7.

33 Dinesh D, Das M, Picado A, Roy L, Rijal S, Singh SP, et al. Insecticide susceptibility of Phlebotomus argentipes in visceral leishmaniasis endemic districts in India and Nepal. PLoS Negl Trop Dis 2010;4:e859.

34 Dinesh DS, Bhattacharya SK, Das P. Peridomestic breeding and resting sites of sandflies (Diptera: Psychodidae) in Bihar, India. Entomol News 2010;120:496-501.

35 Singh R, Lal S, Saxena VK. Breeding ecology of visceral leishmaniasi vector sandfly in Bihar state of India. Acta Trop 2008;107:117-20.

36 Picado A, Kumar V, Das M, Burniston I, Roy L, Suman R, et al. Effect of untreated bed nets on blood-fed Phlebotomus argentipes in kalaazar endemic foci in Nepal and India. Mem Inst Oswaldo Cruz 2009;104:1183-6.

37 Chowdhury R, Huda MM, Kumar V, Das P, Joshi AB, Banjara MJ, et al. The Indian and Nepalese programmes of indoor residual spraying for the elimination of visceral leishmaniasis: performance and effectiveness Ann Trop Med Hyg Parasitol (in press).

38 Courtenay O, Gillingwater K, Gomes PA, Garcez LM, Davies CR. Deltamethrin-impregnated bednets reduce human landing rates of sandfly vector Lutzomyia longipalpis in Amazon households. Med Vet Entomol 2007;21:168-76.

Accepted: 8 October 2010 